\title{
Trastuzumab in the management of gastroesophageal cancer: patient selection and perspectives
}

This article was published in the following Dove Press journal:

OncoTargets and Therapy

25 November 2016

Number of times this article has been viewed

\author{
Michael Davidson \\ Naureen Starling \\ Department of Gastrointestinal \\ Oncology, The Royal Marsden \\ Hospital NHS Foundation Trust, \\ London, UK
}

\begin{abstract}
The addition of trastuzumab to the treatment of a subset of patients with advanced gastric and gastroesophageal junction cancers showing HER2 positivity has been shown to confer clinical benefit; however, questions remain over the optimal methods for defining and selecting such patients. This review provides an overview of current standards for assessing HER2 positivity, the evolving treatment landscape for HER2-positive gastric and esophageal cancers and the challenges and potential future directions in optimal patient selection for HER2-targeted therapy.
\end{abstract}

Keywords: HER2, trastuzumab, gastric cancer, immunohistochemistry, in situ hybridization

\section{Introduction}

Gastroesophageal (GE) cancers are among the leading causes of cancer death worldwide. ${ }^{1}$ The outlook for metastatic disease remains bleak, with median overall survival (OS) generally not reaching more than 1 year in the majority of clinical trials. ${ }^{2}$ The anti-HER2 humanized monoclonal antibody trastuzumab remains a landmark in anticancer drug discovery. It was developed jointly by Genentech and the University of California in the 1990s and gained regulatory approval for breast cancer treatment in 1998. ${ }^{3,4}$ It was later approved in gastric and gastroesophageal junction (GEJ) adenocarcinoma after the ToGA trial showed a survival benefit for a subset of HER2-positive patients in conjunction with first-line chemotherapy. ${ }^{5}$ The only approved indications remain in GE adenocarcinomas and breast cancers; however, there is growing interest in its potential utility for "molecularly triaged" subsets of other solid organ tumors that display HER2 positivity, such as lung and colorectal cancers, although such work remains exploratory. ${ }^{6,7}$ It is generally well tolerated, with commonly reported side effects including flu-like symptoms and mild gastrointestinal upset. ${ }^{8}$ A well-characterized and potentially more serious complication is cardiac dysfunction characterized by decline in ejection fraction. This is a rare and normally reversible side effect, occurring in $2 \%-7 \%$ of patients treated with trastuzumab alone, although the risk is increased when given in conjunction with cardiotoxic chemotherapies. ${ }^{9}$ A number of further HER2-targeting agents have been tested in both early- and late-stage GE cancer with generally disappointing results thus far. ${ }^{10-13}$ Key to the successful clinical application of HER2-directed therapy is appropriate, robust and reproducible patient selection. In the field of gastric cancer, there is some controversy over optimal immunohistochemical (IHC) and in situ hybridization (ISH) genetic analysis, and differing emphasis on the relative importance of protein expression versus gene amplification has led to divergent global standards in the definition of HER2 positivity. This review describes the 
background of HER2 targeting in gastric cancer and discusses some of the current issues and potential future directions in defining and treating HER2-positive patients.

\section{Current treatment landscape}

Treatment for advanced GE cancer remains primarily chemotherapy based; however, the recent regulatory approval of ramucirumab and a growing interest in immunotherapeutic approaches are beginning to expand non-chemotherapy treatment options. There is little consensus on optimal first-line chemotherapy, and standard regimes normally consist of a fluoropyrimidine combined with a platinum agent, with the possible addition of either an anthracycline or a taxane..$^{2,14,15}$ Irinotecan is more commonly used as a second-line therapy; however, several studies have suggested that FOLFIRI (irinotecan with 5-fluorouracil [FU]) also has activity in the first line. ${ }^{16,17}$ Second-line chemotherapy with irinotecan, docetaxel and paclitaxel have all demonstrated a survival advantage over best supportive care (BSC) alone. ${ }^{18-20}$

Phase III trials of ramucirumab have demonstrated significant efficacy in the second-line setting both as monotherapy and in combination with paclitaxel. ${ }^{21,22}$ Evaluation in the first-line setting is ongoing with the phase III RAINFALL study (NCT02314117). As with other cancer types, there is growing interest in immunotherapeutic treatment approaches, primarily focused on the use of checkpoint inhibitors. The KEYNOTE 012 study reported response rates (RRs) of $22 \%$ with pembrolizumab in a heavily pretreated population, and further trials are in progress in both first-line combination with chemotherapy (NCT02335411) and second-line comparison with paclitaxel (NCT02370498). Combination blockade of both PD-1 and VEGFR2 appeared to show a synergistic effect in preclinical models, and a phase I study is currently evaluating the combination of pembrolizumab and ramucirumab (NCT02443324). ${ }^{23}$

\section{HER2 in gastric and esophageal cancers}

HER2 belongs to the EGFR family of transmembrane tyrosine kinase receptors. Unlike other receptors in the family, it has no known activating ligand and must heterodimerize with EGFR, HER3 or HER4 to trigger transphosphorylation and activation of downstream PI3K or MAPK signaling pathways. ${ }^{24}$ When overexpressed, HER2 can homodimerize, giving rise to ligand-independent signaling. ${ }^{25}$ The reported frequency of HER2 overexpression in GE cancer varies widely in the literature, ranging from
$4 \%$ to $>50 \%$ in some reports. ${ }^{26-29}$ The lowest frequency is reported in distal tumors and the highest in tumors of the GEJ. ${ }^{28,29}$ Rates of HER2 expression in squamous cell cancers of the upper esophagus are low, and as a result, the application of HER2-targeted therapy has generally been limited to adenocarcinomas of the lower esophagus and stomach. ${ }^{30}$ An association with intestinal histology has been consistently reported, with HER2 amplification uncommon in diffuse gastric cancers. ${ }^{28}$ A recent case series of 1461 Japanese patients reported an HER2 positivity rate of $21 \%$. Multiple logistic regression analysis identified intestinal type, hepatic metastasis and absence of peritoneal metastasis as significant independent factors related to HER2 positivity. ${ }^{31}$ The association between HER2 expression and prognosis in GE cancer is uncertain; however, a number of studies have now shown HER2 to be a negative prognostic factor associated with more aggressive biological behavior and higher frequencies of recurrence. ${ }^{27,32,33}$ A 2012 systematic review of 42 studies concluded that HER2 positivity was associated with decreased survival and adverse clinicopathological features, including early progression, serosal invasion and more advanced stage. ${ }^{27}$ Such results are consistent with breast cancer, where HER2 positivity is known to be an adverse prognostic factor. ${ }^{34}$

Researchers at The Cancer Genome Atlas (TCGA) recently undertook an analysis of gastric adenocarcinoma utilizing next-generation sequencing (NGS) techniques, describing four distinct subtypes Epstein-Barr virus (EBV)positive tumors, microsatellite-unstable tumors, genomically stable tumors and tumors with chromosomal instability (CIN). ${ }^{35}$ HER 2 amplification was most commonly seen in CIN tumors, with less common prevalence in the EBV-positive and genomically stable subgroups. No microsatellite-unstable tumors were HER2 amplified, although some demonstrated HER 2 missense mutations. The CIN subtype of tumors most associated with HER2 positivity are characterized by CIN and recurrent amplification of other potentially clinically relevant receptor tyrosine kinases. ${ }^{35}$ The interaction between HER2 detection and targeting with other altered tyrosine kinase signaling pathways seen in this subset of tumors is currently unclear.

\section{Defining HER2 positivity}

Standardized protocols for evaluating and defining HER2 positivity were originally developed for breast cancer, with the most widely adopted being the American Society of Clinical Oncology (ASCO)/College of American Pathologists (CAP) clinical practice guidelines. ${ }^{36}$ Testing is through 
either IHC assessment of protein expression using antibody staining or ISH assessment of gene amplification. Traditionally, the most commonly utilized method of gene amplification is fluorescent ISH (FISH), a cytogenetic technique that uses customized fluorescent probes that bind to specific DNA sequences with a high degree of sequence complementarity. ${ }^{37}$ Further emerging techniques include chromogenic and silver-enhanced ISH (CISH/SISH). CISH uses a peroxidase enzyme-labeled probe for chromogenic detection by diaminobenzidine, while SISH uses the same technique with a silverbased detection system. ${ }^{38}$ Because these processes do not involve fluorescent dye, a standard bright-field microscope can be used, circumventing some of the technical difficulties associated with FISH. ${ }^{38}$ An additional technique now becoming more widespread is dual-color, dual-hapten, bright-field ISH (DDISH). This is an automated process that again can be evaluated by conventional microscopy. In contrast to SISH, which requires two separate slides to detect HER2 and CEP17, DDISH uses double-stranded dual-hapten probes to detect both markers on a single slide. Concordance between FISH and DDISH HER 2 amplification results has been found to be high. ${ }^{39}$ Figure 1 illustrates DDISH HER2 positivity in a gastric cancer specimen.

In both breast and GE cancers, protein expression is categorized into IHC $0,1+, 2+$ and $3+$ based upon a score incorporating both the intensity of staining and the percentage or number of cancer cells demonstrating that intensity, however, important differences between the two tumor types have led to modifications in their respective scoring systems. The membranous distribution of protein within breast cancer cells is predominantly circumferential (Figure 2), and breast tumors are defined as IHC $3+$ if there is complete circumferential membrane staining in $>10 \%$ of tumor cells, IHC

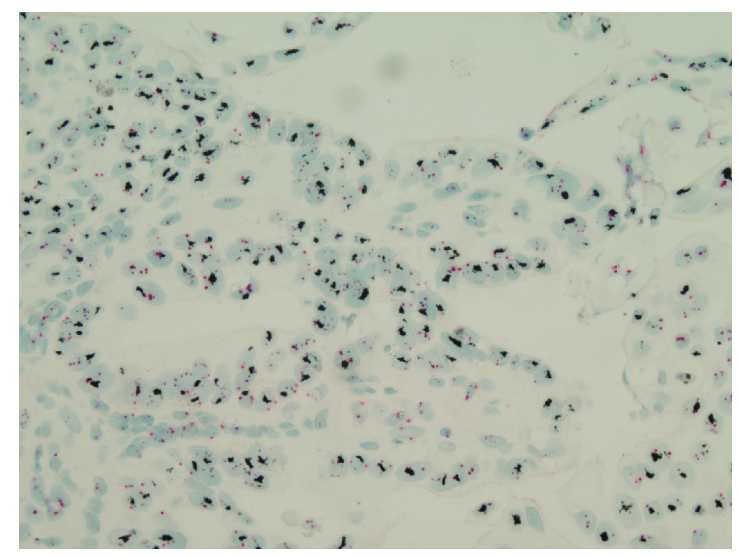

Figure I DDISH evaluation of HER2 amplification in gastric cancer showing both chromosome 17 (red signals) and HER2 probes (black signals).

Abbreviation: DDISH, dual-color, dual-hapten, bright-field in situ hybridization.
$2+$ if there is incomplete circumferential membrane staining in $>10 \%$ or complete staining within $<10 \%$ of cells and IHC $1+$ if there is incomplete faint membrane staining. ${ }^{36}$ HER2 protein expression in GE cancer tends to spare the digestive luminal membrane, resulting in membrane staining that is not completely circumferential but is instead predominantly basolateral or lateral (Figure $3 \mathrm{~A}$ and B).$^{40}$ Furthermore, a greater degree of intratumoral HER2 heterogeneity has been reported in GE specimens, with the presence of variable focal areas of HER2 positivity often seen (Figure 4). ${ }^{41}$

Given these differences, a modified IHC scoring system for gastric cancer was developed for the ToGA trial and validated on 168 specimens in a pre-ToGA study, with high concordance found between the modified IHC criteria and ISH gene amplification results. ${ }^{42}$ This was subsequently validated in a larger dataset and has now been widely adopted by clinicians and institutions worldwide. ${ }^{43}$ The refined GE scoring system does not require complete circumferential homogeneity of staining, and the percentage of cancer cells that are required to show HER 2 expression is lower. A specimen showing weak/moderate or strong complete circumferential "or" basolateral staining is designated as $2+$ or $3+$, respectively. The scoring system also takes into account the more heterogeneous patterns of HER2 positivity in GE cancer by distinguishing between surgical and biopsy specimens. When evaluation is performed on surgical samples, a cutoff point of $10 \%$ of cells in the entire specimen is necessary, whereas for endoscopic biopsy samples a single cluster of at least five positive cells is sufficient. ${ }^{43}$ The sensitivity and specificity of these modified GE criteria for detecting HER2 gene amplification were compared with the ASCO/CAP breast guidelines in a study of 1059 primary gastric adenocarcinomas, which included 123 HER2-amplified tumors. ${ }^{44}$ The modified criteria



Figure 2 HER2 staining in breast cancer illustrating circumferential membranous staining pattern. 

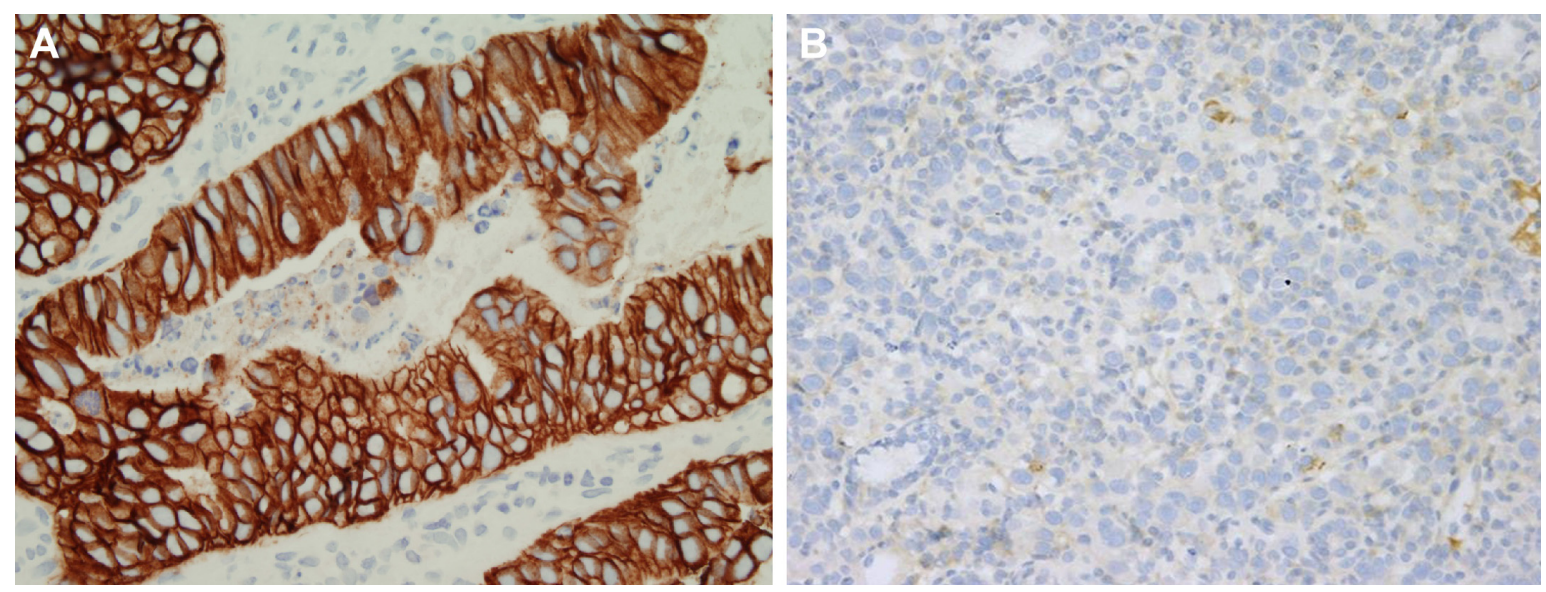

Figure 3 (A) Gastric cancer specimen showing strong staining in >10\% cells (HER2 3+) illustrating predominantly basolateral staining pattern. (B) Gastric cancer specimen showing faint staining in less than $10 \%$ cells (HER2 0 ).

demonstrated significantly higher sensitivity for detecting gene amplification as compared with the breast criteria ( $66 \%$ vs $48 \%$, respectively). Both sets of criteria demonstrated an equally high specificity for correctly identifying negative (IHC 0 and $1+$ ) patients. Formal guidelines for HER2 scoring in gastrointestinal cancer are currently under development by a collaboration between ASCO/CAP and the American Society for Clinical Pathology (ASCP); however, they have not been published at the time of writing. ${ }^{45}$ Comparison of differing HER2 IHC assessment criteria in breast and GE cancers is given in Table 1.

ISH results are reported as the ratio between HER 2 and the chromosome 17 enumeration probe (CEP17) within the nucleus of $\sim 20$ cancer cells. The definition of positivity in both breast and GE cancers is an HER2/CEP17 ratio of $\geq 2$. However, account is also taken of average HER 2 copy number per cell and ISH positivity also includes an HER2/ CEP17 ratio of $<2$ if the HER 2 copy number is $\geq 6$. ISH is equivocal if the HER2/CEP17 ratio is $<2$ with a copy number

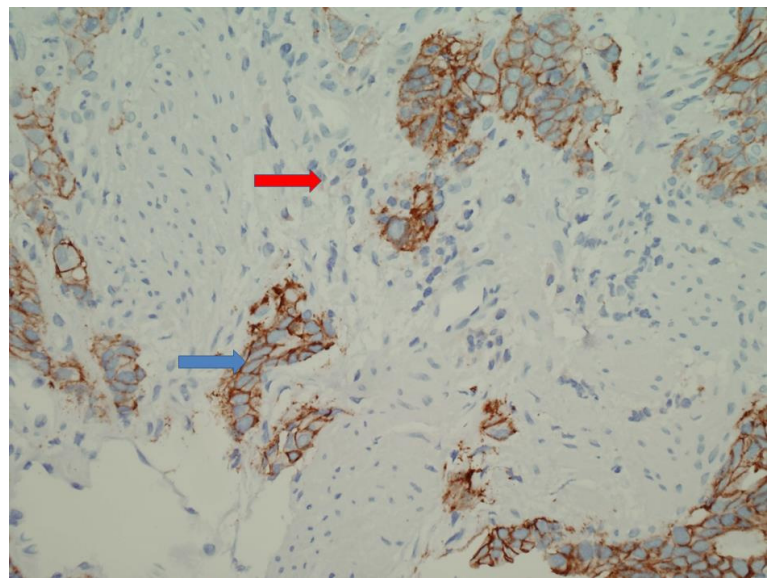

Figure 4 HER2 staining in gastric cancer illustrating tumor heterogeneity within specimen with positively (blue arrow) and negatively (red arrow) stained tumor cells. between 4 and 6 and ISH is negative if the HER2/CEP17 ratio is $<2$ and copy number is $<4 .{ }^{36}$

\section{Clinical role of HER2-directed therapies in the management of gastric and esophageal adenocarcinoma}

\section{First line}

The landmark ToGA trial evaluated the combination of trastuzumab with a cisplatin/fluoropyrimidine chemotherapy doublet in patients with previously untreated, advanced HER2-positive gastric or GEJ adenocarcinoma. ${ }^{5}$ HER2 status was assessed using both IHC and FISH, and tumors were considered HER2 positive on the basis of either an IHC 3+ result or FISH amplification showing an HER2/ CEP17 ratio of $\geq 2$. Among enrolled patients ( $n=594$ of the 3807 screened), almost all tumors were FISH positive, whereas protein expression by IHC varied $(47 \% 3+, 30 \%$ $2+$ and $22 \% 1+$ or 0 ). Toxicity from the combination of the antibody was minimal, and no significant cardiotoxicity was reported. The combination of trastuzumab significantly improved objective RR, progression-free survival (PFS) and OS with the primary end point of median OS improved from 11.1 to 13.8 months. A preplanned exploratory analysis according to HER2 status suggested that OS was improved in patients with high expression of HER2. Patients with the strongest expression (IHC 3+) with concomitant FISH gene amplification received the greatest benefit, with an absolute improvement in OS of $>5$ months (12.3 compared to 17.9 months). IHC expression also appeared to incrementally predict for benefit: trastuzumab was most effective in prolonging survival in IHC $3+$ tumors, less effective in patients with IHC 2+ tumors and ineffective in those with HER2 
Table I Comparative criteria for assessing HER2 protein expression by immunohistochemistry in breast versus GE cancer

\begin{tabular}{|c|c|c|c|c|}
\hline IHC score & Breast: surgical resection or biopsy & GE: surgical resection & GE: biopsy & Assessment \\
\hline $3+$ & $\begin{array}{l}\text { Uniform intense membrane staining in } \\
>10 \% \text { cells }\end{array}$ & $\begin{array}{l}\text { Strong complete, basolateral } \\
\text { or lateral membrane staining } \\
\text { in } \geq 10 \% \text { cells }\end{array}$ & $\begin{array}{l}\text { Strong complete, basolateral } \\
\text { or lateral membrane staining } \\
\text { in a cluster of } \geq 5 \text { tumor cells }\end{array}$ & Positive \\
\hline $2+$ & $\begin{array}{l}\text { Incomplete or weak/moderate circumferential } \\
\text { membrane staining in }>10 \% \text { cells, or } \\
\text { complete and intense circumferential } \\
\text { membrane staining in }<10 \% \text { cells }\end{array}$ & $\begin{array}{l}\text { Weak to moderate } \\
\text { complete, basolateral or } \\
\text { lateral membrane staining in } \\
\geq 10 \% \text { cells }\end{array}$ & $\begin{array}{l}\text { Weak to moderate } \\
\text { complete, basolateral or } \\
\text { lateral membrane staining in } \\
\text { a cluster of } \geq 5 \text { tumor cells }\end{array}$ & Equivocal \\
\hline $\mathrm{I}+$ & $\begin{array}{l}\text { Faint/barely perceptible incomplete } \\
\text { membrane staining in }>10 \% \text { cells }\end{array}$ & $\begin{array}{l}\text { Faint membrane staining } \\
\geq 10 \% \text { cells }\end{array}$ & $\begin{array}{l}\text { Faint membrane staining in a } \\
\text { cluster of } \geq 5 \text { tumor cells }\end{array}$ & Negative \\
\hline 0 & $\begin{array}{l}\text { No staining or faint/barely perceptible } \\
\text { incomplete membrane staining in }<10 \% \text { cells }\end{array}$ & $\begin{array}{l}\text { No staining or staining in } \\
<10 \% \text { cells }\end{array}$ & No staining & Negative \\
\hline
\end{tabular}

Abbreviations: GE, gastroesophageal; IHC, immunohistochemical.

gene-amplified (FISH-positive) but nonprotein-expressing (IHC 0 or 1+) tumors. Some limited phase II evidence has shown that treatment with trastuzumab beyond progression with second-line chemotherapy is a safe and potentially effective treatment option which may have benefit in improving PFS; however, this approach is yet to be validated in any larger-scale clinical trials. ${ }^{46}$

Pertuzumab is a monoclonal antibody directed at the extracellular domain (ECD) of HER2, preventing receptor dimerization. The binding site on domain II is distinct from the trastuzumab binding site on domain IV, allowing the two antibodies to be effectively delivered together. ${ }^{47}$ The combination of pertuzumab with docetaxel and trastuzumab in the first-line treatment of HER2-positive advanced breast cancer improved median PFS by $\sim 6$ months, with little additional toxicity. ${ }^{48} \mathrm{~A}$ phase III evaluation of pertuzumab in combination with first-line trastuzumab with cisplatin/ fluoropyrimidine (the JACOB study, NCT01774786) has been completed, with results anticipated shortly.

\section{Second line}

Trastuzumab-DM1 (T-DM1) is a novel antibody-drug conjugate that combines trastuzumab with a microtubule inhibitor and is effective and well tolerated in previously treated patients with HER2-positive breast cancer. ${ }^{49}$ The GATSBY phase III study evaluated T-DM1 compared with standard taxane therapy for patients with previously treated advanced HER2-positive gastric or GEJ cancer, with a preliminary report at the 2016 ASCO GI Cancers Symposium suggesting a lack of efficacy. ${ }^{11}$ With 415 patients randomized, median OS was 8.6 months with a taxane versus 7.9 months with weekly T-DM1. Grade $\geq 3$ adverse events were numerically lower with T-DM1, and rates of serious adverse events and treatment discontinuations were comparable between both arms.

Lapatinib is an orally available, small-molecule tyrosine kinase inhibitor (TKI) of HER2 and EGFR. In HER2-positive advanced breast cancer, lapatinib is licensed in combination with capecitabine in the second-line setting; however, it is now known to be inferior to single-agent T-DM1. ${ }^{49}$ Singleagent lapatinib demonstrated only modest activity in a phase II study of unselected patients with advanced gastric cancer, but median survival was twice as long in patients with HER2 amplification (defined as gene copy number greater than the median for the cohort) compared with those without. ${ }^{10}$ Two phase III studies of chemotherapy with lapatinib have been conducted. The first-line TRIO-013/LOGiC study failed to demonstrate a significant improvement in OS when lapatinib was combined with a capecitabine/oxaliplatin doublet, although RR and median PFS were significantly improved. ${ }^{12}$ There was no correlation between HER2 IHC score and outcomes. In the second-line TYTAN study, lapatinib combined with paclitaxel was compared with paclitaxel alone. ${ }^{13}$ Again, there was no statistically significant OS benefit. A significant benefit was, however, detected in a preplanned subgroup analysis of patients with the strongest HER2 overexpression (IHC 3+) in whom median survival was almost double compared with chemotherapy alone (14.0 vs 7.6 months), while it was not significantly different in those with IHC $0-2+$ tumors. Selected trials of HER2-targeted agents in the first- and second-line setting are given in Table 2 .

\section{Future directions in HER2 targeting}

In early GE cancer, the phase III ST03 study (NCT00450203) has been adapted to include baseline HER2 testing and randomization of HER2-positive patients to standard perioperative chemotherapy with ECX, or the same therapy along with lapatinib. The randomized phase II INNOVATION trial (NCT02205047) opened in 2015, comparing standard perioperative chemotherapy in patients with resectable gastric cancer combined with trastuzumab or trastuzumab/pertuzumab.

Moving on from the relative disappointment of lapatinib, a number of multi-receptor-targeting TKIs are under 
Table 2 Selected first- and second-line studies of HER2-targeted therapies in GE cancer

\begin{tabular}{|c|c|c|c|c|c|c|}
\hline Trial & Phase & Treatment & $\mathbf{N}$ & ORR (\%) & PFS (months) & OS (months) \\
\hline \multicolumn{7}{|l|}{ First line } \\
\hline \multirow[t]{3}{*}{ ToGA $^{5}$} & III & $\mathrm{CX} / \mathrm{CF}$ & 296 & 47 & 5.5 & 11.1 \\
\hline & & $\mathrm{CX} / \mathrm{CF}+$ trastuzumab $(\mathrm{FISH}$ & $298(131)$ & $35(-)$ & $6.7(-)$ & I3.8 (I7.9) \\
\hline & & positive/HER2 3+ subgroup) & & & & \\
\hline \multirow[t]{2}{*}{ LOGiC $^{12}$} & III & CAPOX + placebo & 273 & 40 & 5.4 & 10.5 \\
\hline & & CAPOX + lapatinib & 272 & 53 & 6.0 & 12.2 \\
\hline HERBIS-166 & $\|^{\#}$ & $\mathrm{SI}+\mathrm{C}+$ trastuzumab & 53 & 68 & 7.8 & 16.0 \\
\hline Grávalos et a $\mathrm{a}^{67}$ & $\mathrm{II}^{\#}$ & $\mathrm{C}+$ trastuzumab & 22 & 32 & 5.1 & - \\
\hline \multicolumn{7}{|l|}{ Second line } \\
\hline \multirow[t]{2}{*}{ GATSBY"I } & $I I / I I I$ & Taxane* & 117 & 21 & 2.9 & 8.6 \\
\hline & & T-DMI & 228 & 20 & 2.7 & 7.9 \\
\hline \multirow[t]{2}{*}{ TYTAN'13 } & III & Paclitaxel & 129 & 9 & 4.4 & 8.9 \\
\hline & & $\begin{array}{l}\text { Paclitaxel + lapatinib (FISH } \\
\text { positive/HER2 3+ subgroup) }\end{array}$ & $132(52)$ & $27(-)$ & $5.4(5.6)$ & $11.0(14.0)$ \\
\hline
\end{tabular}

Notes: \#Non-randomized phase II. *Investigator's choice of either paclitaxel or docetaxel.

Abbreviations: C, cisplatin; CX, cisplatin with capecitabine; CF, cisplatin with 5-FU; CAPOX, capecitabine with oxaliplatin; GE, gastroesophageal; FISH, fluorescent in situ hybridization; 5-FU, 5-fluorouracil; ORR, objective response rate; OS, overall survival; PFS, progression-free survival; T-DMI, trastuzumab-DMI.

investigation in the advanced disease setting. Afatinib, a multi-kinase inhibitor of EGFR, HER2 and HER4, showed signal in a phase II study involving patients with trastuzumab refractory disease. ${ }^{50}$ An expansion cohort looking at safety and efficacy in combination with trastuzumab is currently recruiting (NCT01522768). Neratinib is a further panHER TKI that has shown promising results in trastuzumab refractory HER2-positive breast cancer and is being further investigated in a multi-tumor type phase II study involving solid tumors (including GE cancer) showing HER2, HER3 or EGFR mutations (NCT01953926). ${ }^{51}$ Dacomitinib is an irreversible pan-HER TKI that showed a modest efficacy signal in a phase II monotherapy study; however, no phase III has followed. ${ }^{52}$

A mechanism of action of increasing interest is trastuzumab's immune-mediated antibody-dependent cellular cytotoxicity via activation of antibody-binding Fc receptors. ${ }^{53}$ Murine models have suggested that checkpoint-inhibitor immunotherapy could potentiate these immune-mediated cytotoxic effects, and the combination is being investigated in two clinical trials evaluating trastuzumab with pembrolizumab (NCT02318901) and margetuximab (an Fc-optimized chimeric second-generation anti-HER2 monoclonal antibody) with pembrolizumab (NCT02689284), respectively. ${ }^{54}$

\section{Challenges in HER2 assessment and patient selection \\ Tumor heterogeneity}

The only standardized definition of intratumoral HER2 heterogeneity is the ASCO/CAP breast cancer guidance in which it is defined as the presence of HER2 amplification in 5\%-50\% of analyzed cells. Applying this definition to GE cancer, an HER 2 heterogeneity rate of $17 \%$ has been reported.$^{55}$ However, heterogeneity in GE cancer has been variably defined, leading to large differences in described rates. ${ }^{40,42,56,57}$ The presence of tumor heterogeneity increases the risk of false-negative reporting from biopsy samples, and an expert panel of pathologists working with the ToGA trial has recommended six to eight viable biopsies as necessary for accurate evaluation of HER2 status. ${ }^{58}$ At endoscopy biopsies should preferentially be taken from the lateral surrounds of the tumor as this area has been reported to be more frequently HER2 positive..$^{59}$

Most studies have reported a high concordance between HER2 status in primary tumor and metastatic sites, with a concordance rate that varies between $86 \%$ and $99 \% .{ }^{60-64}$ The GASTHER1 study evaluated the utility of repeat endoscopic biopsy in patients whose tumors were initially found to be HER2 negative (IHC $0-2+$ and ISH negative). ${ }^{65}$ Repeat biopsy identified a "rescue" HER2 positivity rate of $8.7 \%$, with 16 HER2-positive tumors identified out of the 183 retested. Tumors originally classified as IHC $1+$ or $2+$ were more than three times more likely to show HER2 positivity on repeat biopsy than IHC 0 tumors, and liver as a site of metastasis was almost six times more likely to show HER2 positivity than reassessment of other metastatic sites. Patients found to have HER2 positivity on repeat tumor sampling appeared to show similar treatment benefits with trastuzumab to those identified as HER2 positive at initial evaluation. ${ }^{65}$ In contrast to breast cancer, re-biopsy of newly relapsed metastatic sites is not recommended in GE cancer. Data such as these, however, suggest that there may be a role for re-biopsy in carefully considered selected cases. 


\section{$\mathrm{IHC}$ versus gene amplification}

Although chemotherapy with trastuzumab now represents the standard first-line approach for HER2-positive GE cancer, not all patients benefit, with variable RRs of $\sim 30 \%-60 \%$ reported (Table 2). ${ }^{5,66,67}$ Thus, there are a proportion of patients who do not respond to trastuzumab therapy, despite their cancer conventionally being defined as HER2 positive. The correlation between the magnitude of HER 2 gene amplification and trastuzumab sensitivity has not been well evaluated in GE cancer, with only two exploratory reports on the topic published to date. In a 2013 article, Gomez-Martin et al ${ }^{68}$ suggested that HER2 gene amplification was a predictive factor for trastuzumab sensitivity. A total of 90 patients with metastatic gastric cancer treated with first-line chemotherapy plus trastuzumab were studied, and optimal HER2/CEP17 cutoff values for discriminating positive results in terms of response and survival were determined using receiver operating characteristic (ROC) curve analyses. An HER2/ CEP17 ratio of 4.7 was identified as the optimal cutoff value discriminating for response. A more recent 2015 study also investigated the association between trastuzumab efficacy and $H E R 2$ gene amplification and compared its predictability according to IHC status. ${ }^{69} \mathrm{~A}$ total of 126 patients treated with chemotherapy plus trastuzumab were enrolled and HER2 IHC score, HER2/CEP17 ratio and HER 2 gene copy number were analyzed. Patients with IHC $3+$ showed significantly longer OS than patients with IHC $\leq 2+$, and an HER2/CEP17 ratio of 4.48 was found to be the optimal cutoff for predicting for survival (26.9 vs 14.7 months). In a subgroup analysis, the treatment outcome of patients with IHC 3+ was not influenced by the level of HER2 gene amplification, suggesting that further gene amplification testing for this group of patients does not provide additional predictive value for clinical outcome. However, in patients with IHC $\leq 2+$, both HER2/ CEP17 ratio and HER2 gene copy number were informative, with an HER2/CEP1 7 ratio of $>3.69$ and an HER 2 gene copy number of $>7.75$ predicting for better outcomes. It is notable that these cutoffs are higher than the current standard FISHpositivity cutoffs of 2 and 6 , respectively.

There are high levels of concordance between HER2 gene amplification and the highest and lowest protein expression groups, with reported ISH positivity rates of $88 \%-100 \%$ for IHC $3+$ tumors and $0 \%-8 \%$ for IHC $0-1+$ tumors. ${ }^{44,56,70}$ ToGA screening data show that of those who were classified as IHC $3+, 94.9 \%$ also showed gene amplification by FISH. ${ }^{56}$ The relationship between intermediate IHC $2+$ protein expression and gene amplification, however, is less clear. Of the patients with IHC $2+$ identified from
ToGA screening, $54.6 \%$ were FISH amplified. There are no existing data to suggest mechanisms other than HER2 gene amplification to explain the overexpression of the HER2 protein. Variable results of IHC evaluation have been reported dependent upon the IHC techniques used, and low IHC results in the presence of amplification may be caused by variability in operator-dependent IHC staining evaluation..$^{71}$ The known tumor heterogeneity in GE cancer may also contribute, as areas lacking HER2 overexpression often exhibit HER 2 amplification. ${ }^{71}$ In cases of equivocal or uncertain HER2 expression, the use of amplification level as a continuous biomarker may potentially prove to be a more robust and rational approach for selecting patients likely to benefit from anti-HER2 therapies.

\section{Cardiac risk}

There is a lack of consensus, evidence-based guidance for the identification, treatment and follow-up of trastuzumabinduced cardiac dysfunction, with wide variation in practice globally. ${ }^{72}$ Similarly, there are no published guidelines for the exclusion of patients with GE cancer for trastuzumab on the basis of excess cardiac risk, and many clinicians use the cardiac eligibility criteria from the main adjuvant trastuzumab trials in breast cancer. These were also adopted by the ToGA investigators and include congestive heart failure, baseline left ventricular ejection fraction (LVEF) $<50 \%$, transmural myocardial infarction, poorly controlled hypertension (>180/100), angina pectoris requiring medication, clinically significant valvular heart disease and high-risk arrhythmias as contraindications to treatment. ${ }^{5}$ Cardiac monitoring recommendations for patients with GE cancer also generally follow those established for breast cancer, and the UK National Institute for Health and Care Excellence (NICE) guidance is representative, recommending assessment at three monthly intervals with either ECHO or multigated acquisition (MUGA) scan. If the LVEF drops by 10 percentage points or more from baseline and to below $50 \%$, then trastuzumab treatment should be stopped, with a decision to resume based on further cardiac assessment and an informed consideration of individual risk versus benefit on a case-by-case basis. ${ }^{73}$ The majority of trastuzumab-induced cardiac dysfunction occurs during the first 6 months of treatment and onset is rare during treatment beyond 6 months or after treatment cessation, thus less frequent monitoring may be justified..$^{74}$ Defining cardiac dysfunction through LVEF assessment - particularly with the use of MUGA scanning only - may lack sensitivity to detect early preclinical changes and other more subtle wall motion and valvular pathologies. ${ }^{72}$ 


\section{Current selection standards}

Guidelines for selecting patients with GE cancer for trastuzumab treatment based upon HER2 testing are not uniform worldwide. The ToGA study led to rapid regulatory approval, with the US Food and Drug Administration (FDA) approving the drug for patients with IHC 3+ HER2 overexpression or FISH-confirmed gene amplification (with any IHC expression), in keeping with the study eligibility criteria. The European Medicines Agency (EMEA), however, approved the drug in patients with IHC $3+$, or IHC $2+$ and FISHamplified cancers, a subgroup that was assessed in the study only as part of an exploratory analysis. In the UK, the NICE guidelines recommend the drug for the higher threshold of HER2 IHC $3+$ tumors only. The $22 \%$ of FISH-positive and IHC $0-1+$ patients in ToGA did not gain significant clinical benefit. This suggests that screening for HER2 status by initial FISH testing may result in a proportion of nonresponders being exposed to both the risk and expense of trastuzumab unnecessarily. As a result, the EMEA has recommended that the initial screening test for HER2 should be with IHC, followed by confirmatory FISH testing for IHC $2+$ cases only. ${ }^{75}$ Conversely, in the UK, NICE guidance mandating treatment for IHC 3+ patients only and not taking into account gene amplification is almost certainly depriving trastuzumab from a subset of patients who would benefit.

\section{Future perspectives: circulating biomarkers}

Multiple studies have addressed whether circulating HER2 protein ECD levels (c-ECD) can predict outcome with HER2directed treatment. ${ }^{76,77}$ Baseline c-ECD levels do not appear to predict for response, but in a pooled analysis of seven trials of first-line trastuzumab with or without chemotherapy in patients with breast cancer, those with a $20 \%$ or greater decline in c-ECD levels over baseline had significantly higher RRs, PFS and OS compared with those with a lesser degree of decline. ${ }^{77}$ However, a separate review of 63 studies concluded that concentrations of HER2 c-ECD are not consistently related to patient outcomes. ${ }^{78}$ Thus, the clinical utility of assessing or following c-ECD levels during HER2directed therapy is not established and the ASCO expert panel on tumor markers in breast cancer has recommended against its use in any clinical setting.

Digital polymerase chain reaction (PCR) has the potential to accurately quantify the concentration of nucleic acids in a sample to a much greater degree than traditional quantitative PCR by counting individual DNA molecules. ${ }^{79}$ The procedure requires small concentrations of DNA for accurate analysis and thus is well suited to identifying genetic amplifications in circulating tumor (ct)DNA, an area under considerable study across multiple tumor types. ${ }^{80}$ In breast cancer, an analysis of ctDNA with digital PCR reported high accuracy in the determination of HER 2 status, with $90 \%$ concordance with tumor-derived HER 2 status in an independent validation set. ${ }^{81}$ In gastric cancer, digital droplet (dd)PCR was used to evaluate HER2 amplification in both biopsy specimens and circulating tumor (ct)DNA in 25 patients. ${ }^{82}$ The concordance rate between tumor analysis with ddPCR and IHC/FISH was high at $92 \%$. The concordance rate with ctDNA was lower (62.5\%); however, patients who were HER2 positive by ctDNA had significantly shorter survival compared with HER2-negative patients, suggesting a potential prognostic role. A further pilot study has evaluated the ability of ctDNA ddPCR to reflect dynamic changes in HER2 during gastric cancer treatment and follow-up. ${ }^{83} \mathrm{~A}$ total of 60 patients with gastric cancer undergoing surgery were followed up with sequential analysis of ctDNA. Preoperative HER2 ratio in ctDNA correlated well with tumor HER2 status and high plasma HER 2 ratios were identified in seven out of 13 patients at a time point of relapse after prior radical surgery. This very early data suggest some promise for the use of ddPCR HER2 assessment as a repeatable and noninvasive approach for real-time evaluations of HER2 status during treatment; however, more work in the area is required.

\section{Discussion}

Trastuzumab has been an exemplar for biomarker-driven, targeted treatment based on individual tumor biology in GE cancer. There remain significant challenges, however, in leveraging maximum clinical benefit for patients in a resourceefficient manner. Uncertainties remain over the optimum definition of HER2 positivity and the mandating of ISH positivity in IHC $2+$ equivocal cases only would seem to be more in-keeping with the patterns of clinical benefit seen in the ToGA study. It is interesting that both recently reported studies investigating the relationship between HER2 gene amplification and clinical benefit from trastuzumab came to very similar conclusions regarding the optimal HER2/CEP17 ratio predicting for trastuzumab benefit, which was considerably higher than the currently mandated FISH definition of positivity. ${ }^{68,69}$ Further attempts to target HER2 amplification such as with the antibody-drug conjugate T-DM1 and the small molecule inhibitor lapatinib have been disappointing. Again, however, data from lapatinib studies suggest that it may have a role in a subgroup of strongly HER2-expressing patients. ${ }^{10,13}$

Heterogeneity of HER2 expression in GE cancer is a challenging but not an insurmountable problem. Endoscopic biopsies, if performed and analyzed to existing standards, 
can provide reliable HER2 assessment. ${ }^{58}$ There is relative consistency between primary GE tumors and metastatic sites, suggesting that, unlike breast cancer, routine re-biopsy of all newly relapsed metastatic sites would not be appropriate. The GASTHER1 data, however, suggest that in carefully selected cases, such as those tumors originally showing IHC $1+$ or $2+$ expression and where the relapsed site is in the liver, re-biopsy may be indicated to pick up discordant emergent HER2 positivity. ${ }^{65}$

The use of circulating blood biomarkers as a measure of HER2 positivity and dynamic response to treatment is still very much at an early stage, and whether this results in meaningful clinical application has yet to be seen. Such circulating biomarkers may offer a noninvasive method of ascertaining HER2 status at baseline, while dynamic changes in HER2 expression during treatment may shed light on resistance mechanisms to HER2-targeted therapy, which are poorly understood at present. Moving forward, it is hoped that a greater understanding of the relationship between HER2 protein expression, gene amplification and blood-borne biomarkers with treatment response will allow for more individualized medical decisions regarding the application of HER2 therapies in GE cancer to be made.

\section{Acknowledgment}

MD and NS would like to thank Dr Andrew Wotherspoon for critically reviewing the manuscript and providing the images.

\section{Disclosure}

The authors report no conflicts of interest in this work.

\section{References}

1. Ferlay J, Soerjomataram I, Ervik M, Forman D, Bray F. GLOBOCAN 2012 v1.0, Cancer Incidence and Mortality Worldwide. IARC CancerBase. Lyon, France: International Agency for Research on Cancer; 2014.

2. Wagner AD, Unverzagt $\mathrm{S}$, Grothe $\mathrm{W}$, et al. Chemotherapy for advanced gastric cancer. Cochrane Database Syst Rev. 2010;3(3):CD004064.

3. Slamon DJ, Leyland-Jones B, Shak S, et al. Use of chemotherapy plus a monoclonal antibody against HER2 for metastatic breast cancer that overexpresses HER2. N Engl J Med. 2001;344(11):783-792.

4. Smith I, Procter M, Gelber RD, et al. 2-year follow-up of trastuzumab after adjuvant chemotherapy in HER2-positive breast cancer: a randomised controlled trial. Lancet. 2007;369(9555):29-36.

5. Bang YJ, Van Cutsem E, Feyereislova A, et al. Trastuzumab in combination with chemotherapy versus chemotherapy alone for treatment of HER2-positive advanced gastric or gastro-oesophageal junction cancer (ToGA): a phase 3, open-label, randomised controlled trial. Lancet. 2010;376(9742):687-697.

6. Mazières J, Peters S, Lepage B, et al. Lung cancer that harbors an HER2 mutation: epidemiologic characteristics and therapeutic perspectives. J Clin Oncol. 2013;31(16):1997-2003.

7. Sartore-Bianchi A, Trusolino L, Martino C, et al. Dual-targeted therapy with trastuzumab and lapatinib in treatment-refractory, KRAS codon 12/13 wild-type, HER2-positive metastatic colorectal cancer (HERACLES): a proof-of-concept, multicentre, open-label, phase 2 trial. Lancet Oncol. 2016;17(6):738-746.
8. Herceptin [webpage on the Internet]. Summary of Product Characteristics. Available from: https://www.nice.org.uk/guidance/ta107/documents/ summary-of-product-characteristics2. Accessed October 26, 2016.

9. Seidman A, Hudis C, Pierri MK, et al. Cardiac dysfunction in the trastuzumab clinical trials experience. J Clin Oncol. 2002;20(5):1215-1221.

10. Iqbal S, Goldman B, Fenoglio-Preiser CM, et al. Southwest Oncology Group study S0413: a phase II trial of lapatinib (GW572016) as firstline therapy in patients with advanced or metastatic gastric cancer. Ann Oncol. 2011;22(12):2610-2615.

11. Kang Y-K, Shah M, Ohtsu A, Van Cutsem E. A randomized, openlabel, multicenter, adaptive phase $2 / 3$ study of trastuzumab emtansine (T-DM1) versus a taxane (TAX) in patients (pts) with previously treated HER2-positive locally advanced or metastatic gastric/gastroesophageal junction adenocarcinoma. J Clin Oncol. 2016;34(suppl 4s):Abstr5.

12. Hecht JR, Bang Y-J, Qin SK, et al. Lapatinib in combination with capecitabine plus oxaliplatin in human epidermal growth factor receptor 2-positive advanced or metastatic gastric, esophageal, or gastroesophageal adenocarcinoma: TRIO-013/LOGiC - a randomized phase III trial. J Clin Oncol. 2016;34(5):443-451.

13. Satoh T, Doi T, Ohtsu A, et al. Lapatinib plus paclitaxel versus paclitaxel alone in the second-line treatment of HER2-amplified advanced gastric cancer in Asian populations: TyTAN - a randomized, phase III study. J Clin Oncol. 2014;32(19):2039-2049.

14. Cunningham D, Starling N, Rao S, et al. Capecitabine and oxaliplatin for advanced esophagogastric cancer. $N$ Engl J Med. 2008;358(1):36-46.

15. Van Cutsem E, Moiseyenko VM, Tjulandin S, et al. Phase III study of docetaxel and cisplatin plus fluorouracil compared with cisplatin and fluorouracil as first-line therapy for advanced gastric cancer: a report of the V325 Study Group. J Clin Oncol. 2006;24(31):4991-4997.

16. Dank M, Zaluski J, Barone C, et al. Randomized phase III study comparing irinotecan combined with 5 -fluorouracil and folinic acid to cisplatin combined with 5 -fluorouracil in chemotherapy naive patients with advanced adenocarcinoma of the stomach or esophagogastric junction. Ann Oncol. 2008;19(8):1450-1457.

17. Guimbaud R, Louvet C, Ries P, et al. Prospective, randomized, multicenter, phase III study of fluorouracil, leucovorin, and irinotecan versus epirubicin, cisplatin, and capecitabine in advanced gastric adenocarcinoma: a French intergroup (Fédération Francophone de Cancérologie Digestive, Féd. J Clin Oncol. 2014;32(31):3520-3526.

18. Thuss-Patience PC, Kretzschmar A, Bichev D, et al. Survival advantage for irinotecan versus best supportive care as second-line chemotherapy in gastric cancer - a randomised phase III study of the Arbeitsgemeinschaft Internistische Onkologie (AIO). Eur J Cancer. 2011;47(15): 2306-2314.

19. Kang JH, Lee SI, Lim do H, et al. Salvage chemotherapy for pretreated gastric cancer: a randomized phase III trial comparing chemotherapy plus best supportive care with best supportive care alone. J Clin Oncol. 2012;30(13):1513-1518.

20. Ford HE, Marshall A, Bridgewater JA, et al. Docetaxel versus active symptom control for refractory oesophagogastric adenocarcinoma (COUGAR-02): an open-label, phase 3 randomised controlled trial. Lancet Oncol. 2014;15(1):78-86.

21. Fuchs CS, Tomasek J, Yong CJ, et al. Ramucirumab monotherapy for previously treated advanced gastric or gastro-oesophageal junction adenocarcinoma (REGARD): an international, randomised, multicentre, placebo-controlled, phase 3 trial. Lancet. 2014;383(9911):31-39.

22. Wilke H, Muro K, Van Cutsem E, et al. Ramucirumab plus paclitaxel versus placebo plus paclitaxel in patients with previously treated advanced gastric or gastro-oesophageal junction adenocarcinoma (RAINBOW): a double-blind, randomised phase 3 trial. Lancet Oncol. 2014;15: 1224-1235.

23. Yasuda S, Sho M, Yamato I, et al. Simultaneous blockade of programmed death 1 and vascular endothelial growth factor receptor 2 (VEGFR2) induces synergistic anti-tumour effect in vivo. Clin Exp Immunol. 2013; 172(3):500-506.

24. Daly JM, Olayioye MA, Wong AM, et al. NDF/heregulin-induced cell cycle changes and apoptosis in breast tumour cells: role of PI3 kinase and p38 MAP kinase pathways. Oncogene. 1999;18(23):3440-3451. 
25. Worthylake R, Opresko LK, Wiley HS. ErbB-2 amplification inhibits down-regulation and induces constitutive activation of both ErbB-2 and epidermal growth factor receptors. J Biol Chem. 1999;274(13): 8865-8874.

26. Kim KC, Koh YW, Chang H-M, et al. Evaluation of HER2 protein expression in gastric carcinomas: comparative analysis of 1,414 cases of whole-tissue sections and 595 cases of tissue microarrays. Ann Surg Oncol. 2011;18(10):2833-2840.

27. Jørgensen JT, Hersom M. HER2 as a prognostic marker in gastric cancera systematic analysis of data from the literature. J Cancer. 2012;3: 137-144.

28. Koopman T, Smits MM, Louwen M, Hage M, Boot H, Imholz ALT. HER2 positivity in gastric and esophageal adenocarcinoma: clinicopathological analysis and comparison. J Cancer Res Clin Oncol. 2015; 141(8):1343-1351.

29. Kim W-H, Gomez-Izquierdo L, Vilardell F, et al. HER2 status in gastric and gastroesophageal junction cancer: results of the large, multinational HER-EAGLE study. Appl Immunohistochem Mol Morphol. Epub 2016 Aug 3.

30. Huang J-X, Zhao K, Lin M, et al. HER2 gene amplification in esophageal squamous cell carcinoma is less than in gastroesophageal junction and gastric adenocarcinoma. Oncol Lett. 2013;6(1):13-18.

31. Matsusaka S, Nashimoto A, Nishikawa K, et al. Clinicopathological factors associated with HER2 status in gastric cancer: results from a prospective multicenter observational cohort study in a Japanese population (JFMC44-1101). Gastric Cancer. 2016;19(3):839-851.

32. Baykara M, Benekli M, Ekinci O, et al. Clinical significance of HER2 overexpression in gastric and gastroesophageal junction cancers. J Gastrointest Surg. 2015;19(9):1565-1571.

33. Kurokawa Y, Matsuura N, Kimura Y, et al. Multicenter large-scale study of prognostic impact of HER2 expression in patients with resectable gastric cancer. Gastric Cancer. 2015;18(4):691-697.

34. Gusterson BA, Gelber RD, Goldhirsch A, et al. Prognostic importance of c-erbB-2 expression in breast cancer. International (Ludwig) Breast Cancer Study Group. J Clin Oncol. 1992;10(7):1049-1056.

35. Bass AJ, Thorsson V, Shmulevich I, et al. Comprehensive molecular characterization of gastric adenocarcinoma. Nature. 2014;513(7517): 202-209.

36. Wolff AC, Hammond MEH, Hicks DG, et al. Recommendations for human epidermal growth factor receptor 2 testing in breast cancer: American Society of Clinical Oncology/College of American Pathologists clinical practice guideline update. J Clin Oncol. 2013;31(31):3997-4013.

37. Pala EE, Bayol U, Ozguzer A, Akman O. HER2 status in gastric cancer: a comparison of two novel in situ hybridization methods (IQ FISH and dual color SISH) and two immunohistochemistry methods (A0485 and HercepTest $^{\mathrm{TM}}$ ). Pathol Res Pract. 2013;209(9):548-554.

38. Penault-Llorca F, Bilous M, Dowsett M, et al. Emerging technologies for assessing HER2 amplification. Am J Clin Pathol. 2009;132(4): $539-548$.

39. Kosa C, Kardos L, Kovacs J, Szollosi Z. Comparison of dual-color dual-hapten brightfield in situ hybridization (DDISH) and fluorescence in situ hybridization in breast cancer HER2 assessment. Pathol Res Pract. 2013;209(3):147-150.

40. Lee HE, Park KU, Yoo SB, et al. Clinical significance of intratumoral HER2 heterogeneity in gastric cancer. Eur J Cancer. 2013;49(6):1448-1457.

41. Vance GH, Barry TS, Bloom KJ, et al. Genetic heterogeneity in HER2 testing in breast cancer: panel summary and guidelines. Arch Pathol Lab Med. 2009;133(4):611-612.

42. Hofmann M, Stoss O, Shi D, et al. Assessment of a HER2 scoring system for gastric cancer: results from a validation study. Histopathology. 2008;52(7):797-805.

43. Rüschoff J, Dietel M, Baretton G, et al. HER2 diagnostics in gastric cancer-guideline validation and development of standardized immunohistochemical testing. Virchows Arch. 2010;457(3):299-307.

44. Park YS, Hwang HS, Park HJ, et al. Comprehensive analysis of HER2 expression and gene amplification in gastric cancers using immunohistochemistry and in situ hybridization: which scoring system should we use? Hum Pathol. 2012;43(3):413-422.
45. ASCO [webpage on the Internet]. CAP, ASCP, ASCO Invite Public Comment on Gastric Cancer Guideline. Multidisciplinary Guidance Document Focuses on HER2 Testing for Patients with Gastric or Gastroesophageal Cancer. 2015. Available from: https:// www.asco.org/about-asco/press-center/news-releases/cap-ascpasco-invite-public-comment-gastric-cancer-guideline. Accessed August 15, 2016.

46. Li Q, Jiang $\mathrm{H}$, Li H, et al. Efficacy of trastuzumab beyond progression in HER2 positive advanced gastric cancer: a multicenter prospective observational cohort study. Oncotarget. 2. Epub 2016 Jul 7.

47. Richard S, Selle F, Lotz J-P, Khalil A, Gligorov J, Soares DG. Pertuzumab and trastuzumab: the rationale way to synergy. An Acad Bras Ciênc. 2016;88(suppl 1):565-577.

48. Baselga J, Cortés J, Kim S-B, et al. Pertuzumab plus trastuzumab plus docetaxel for metastatic breast cancer. $N$ Engl J Med. 2012;366(2): 109-119.

49. Verma S, Miles D, Gianni L, et al. Trastuzumab emtansine for HER2-positive advanced breast cancer. $N$ Engl J Med. 2012;367(19): 1783-1791.

50. Janjigian YY, Ku G, Ilson D, Boyal M. A phase II study of afatinib in patients (pts) with metastatic human epidermal growth factor receptor (HER2)-positive trastuzumab refractory esophagogastric (EG) cancer. 2015 Gastrointestinal Cancers Symposium Abstracts Meeting Library. J Clin Oncol. 2015;33(suppl 3):abstr59.

51. Burstein HJ, Sun Y, Dirix LY, et al. Neratinib, an irreversible ErbB receptor tyrosine kinase inhibitor, in patients with advanced ErbB2positive breast cancer. J Clin Oncol. 2010;28(8):1301-1307.

52. Oh D-Y, Lee K-W, Cho JY, Kang WK. A phase II open-label trial of dacomitinib monotherapy in patients with HER2-positive advanced gastric cancer after failure of at least one prior chemotherapy regimen. 2012 Gastrointestinal Cancers Symposium Abstracts Meeting Library. J Clin Oncol. 2012;30(suppl 4):abstr54.

53. Arnould L, Gelly M, Penault-Llorca F, et al. Trastuzumab-based treatment of HER2-positive breast cancer: an antibody-dependent cellular cytotoxicity mechanism? Br J Cancer. 2006;94(2):259-267.

54. Stagg J, Loi S, Divisekera U, et al. Anti-ErbB-2 mAb therapy requires type I and II interferons and synergizes with anti-PD-1 or anti-CD137 mAb therapy. Proc Natl Acad Sci U S A. 2011;108(17):7142-7147.

55. Yoon HH, Shi Q, Sukov WR, et al. Adverse prognostic impact of intratumor heterogeneous HER2 gene amplification in patients with esophageal adenocarcinoma. J Clin Oncol. 2012;30(32):3932-3938.

56. Van Cutsem E, Bang Y-J, Feng-Yi F, et al. HER2 screening data from ToGA: targeting HER2 in gastric and gastroesophageal junction cancer. Gastric Cancer. 2015;18(3):476-484.

57. Ahn S, Ahn S, Van Vrancken M, et al. Ideal number of biopsy tumor fragments for predicting HER2 status in gastric carcinoma resection specimens. Oncotarget. 2015;6(35):38372-38380.

58. Rüschoff J, Hanna W, Bilous M, et al. HER2 testing in gastric cancer: a practical approach. Mod Pathol. 2012;25(5):637-650.

59. Tominaga N, Gotoda $T$, Hara M, et al. Five biopsy specimens from the proximal part of the tumor reliably determine HER2 protein expression status in gastric cancer. Gastric Cancer. 2016;19(2):553-560.

60. Marx AH, Tharun L, Muth J, et al. HER-2 amplification is highly homogenous in gastric cancer. Hum Pathol. 2009;40(6):769-777.

61. Bozzetti C, Negri FV, Lagrasta CA, et al. Comparison of HER2 status in primary and paired metastatic sites of gastric carcinoma. $\mathrm{Br}$ J Cancer. 2011;104(9):1372-1376.

62. Kim MA, Lee H-J, Yang H-K, Bang Y-J, Kim WH. Heterogeneous amplification of ERBB2 in primary lesions is responsible for the discordant ERBB2 status of primary and metastatic lesions in gastric carcinoma. Histopathology. 2011;59(5):822-831.

63. Cho EY, Park K, Do I, et al. Heterogeneity of ERBB2 in gastric carcinomas: a study of tissue microarray and matched primary and metastatic carcinomas. Mod Pathol. 2013;26(5):677-684.

64. Grillo F, Fassan M, Ceccaroli C, et al. The reliability of endoscopic biopsies in assessing HER2 status in gastric and gastroesophageal junction cancer: a study comparing biopsies with surgical samples. Transl Oncol. 2013;6(1):10-16. 
65. Park SR, Park YS, Ryu M-H, et al. Extra-gain of HER2-positive cases through HER2 reassessment in primary and metastatic sites in advanced gastric cancer with initially HER2-negative primary tumours: results of GASTric cancer HER2 reassessment study 1 (GASTHER1). Eur $J$ Cancer. 2016;53:42-50.

66. Kurokawa Y, Sugimoto N, Miwa H, et al. Phase II study of trastuzumab in combination with S-1 plus cisplatin in HER2-positive gastric cancer (HERBIS-1). Br J Cancer. 2014;110(5):1163-1168.

67. Grávalos C, Gómez-Martín C, Rivera F, et al. Phase II study of trastuzumab and cisplatin as first-line therapy in patients with HER2-positive advanced gastric or gastroesophageal junction cancer. Clin Transl Oncol. 2011;13(3):179-184.

68. Gomez-Martin C, Plaza JC, Pazo-Cid R, et al. Level of HER2 gene amplification predicts response and overall survival in HER2-positive advanced gastric cancer treated with trastuzumab. J Clin Oncol. 2013;31(35): 4445-4452.

69. Ock C-Y, Lee K-W, Kim JW, et al. Optimal patient selection for trastuzumab treatment in HER2-positive advanced gastric cancer. Clin Cancer Res. 2015;21(11):2520-2529.

70. Yoon HH, Sukov WR, Shi Q, et al. HER-2/neu gene amplification in relation to expression of HER2 and HER3 proteins in patients with esophageal adenocarcinoma. Cancer. 2014;120(3):415-424.

71. Kunz PL, Mojtahed A, Fisher GA, et al. HER2 expression in gastric and gastroesophageal junction adenocarcinoma in a US population: clinicopathologic analysis with proposed approach to HER2 assessment. Appl Immunohistochem Mol Morphol. 2012;20(1):13-24.

72. Hamo CE, Bloom MW, Cardinale D, et al. Cancer therapy-related cardiac dysfunction and heart failure. Circ Heart Fail. 2016;9(2): e002843.

73. NICE [webpage on the Internet]. Trastuzumab for the Adjuvant Treatment of Early-Stage HER2-Positive Breast Cancer NICE Technology Appraisal Guidance [TA107]. 2006. Available from: https:/www.nice.org.uk/ guidance/TA107/chapter/1-guidance. Accessed August 15, 2016.
74. Davis CC,Zelnak A, Eley JW, Goldstein DA, Switchenko JM, McKibbin T. Clinical utility of routine cardiac monitoring in breast cancer patients receiving trastuzumab. Ann Pharmacother. 2016;50(9):712-717.

75. European Medicines Agency. Committee for Medicinal Products for Human Use Post-Authorisation Summary of Positive Opinion for Herceptin. 2009. Available from: http://www.ema.europa.eu/docs/ en_GB/document_library/Summary_of_opinion/human/000278/ WC500059913.pdf. Accessed August 10, 2016.

76. Fornier MN, Seidman AD, Schwartz MK, et al. Serum HER2 extracellular domain in metastatic breast cancer patients treated with weekly trastuzumab and paclitaxel: association with HER2 status by immunohistochemistry and fluorescence in situ hybridization and with response rate. Ann Oncol. 2005;16(2):234-239.

77. Ali SM, Carney WP, Esteva FJ, et al. Serum HER-2/neu and relative resistance to trastuzumab-based therapy in patients with metastatic breast cancer. Cancer. 2008;113(6):1294-1301.

78. Leyland-Jones B, Smith BR, Alroy I, et al. Serum HER2 testing in patients with HER2-positive breast cancer: the death knell tolls. Lancet Oncol. 2011;12(3):286-295.

79. Vogelstein B, Kinzler KW. Digital PCR. Proc Natl Acad Sci US A. 1999; 96(16):9236-9241.

80. Hudecova I. Digital PCR analysis of circulating nucleic acids. Clin Biochem. 2015;48(15):948-956.

81. Gevensleben H, Garcia-Murillas I, Graeser MK, et al. Noninvasive detection of HER2 amplification with plasma DNA digital PCR. Clin Cancer Res. 2013;19(12):3276-3284.

82. Kinugasa H, Nouso K, Tanaka T, et al. Droplet digital PCR measurement of HER2 in patients with gastric cancer. Br J Cancer. 2015;112(10): 1652-1655.

83. Shoda K, Ichikawa D, Fujita Y, et al. Monitoring the HER2 copy number status in circulating tumor DNA by droplet digital PCR in patients with gastric cancer. Gastric Cancer. Epub 2016 Feb 13.
OncoTargets and Therapy

\section{Publish your work in this journal}

OncoTargets and Therapy is an international, peer-reviewed, open access journal focusing on the pathological basis of all cancers, potential targets for therapy and treatment protocols employed to improve the management of cancer patients. The journal also focuses on the impact of management programs and new therapeutic agents and protocols on

\section{Dovepress}

patient perspectives such as quality of life, adherence and satisfaction. The manuscript management system is completely online and includes a very quick and fair peer-review system, which is all easy to use. Visit http://www.dovepress.com/testimonials.php to read real quotes from published authors. 